\title{
Overexpression of heparanase in ovarian cancer and its clinical significance
}

\author{
WEI ZHANG, HONG CHAN, LOUWEI WEI, ZHONGMIAN PAN, JIEQING ZHANG and LI LI \\ Department of Gynecologic Oncology, Affiliated Tumor Hospital of Guangxi Medical University, \\ Nanning, Guangxi 530021, P.R. China
}

Received June 12, 2013; Accepted August 1, 2013

DOI: $10.3892 /$ or.2013.2701

\begin{abstract}
It has been reported that heparanase (HPSE) is overexpressed in ovarian cancer and is associated with tumor invasion and metastasis. However, a systematic study on the contribution of HPSE to tumor metastasis is rarely reported. In this study, based on the measurement of HPSE serum concentration, the expression of HPSE at both the mRNA and protein levels in tumors and its effects on the biological behaviors of cancer cells, we elucidated the role of HPSE in tumor invasion and metastasis in ovarian cancer and concluded that either the expression of HPSE in cancer and/or the serum concentration of HPSE may be a useful biomarker for the evaluation of surgery effects and prognosis prediction.
\end{abstract}

\section{Introduction}

Ovarian cancer is the most fatal cancer of the female reproductive system and a cause of high mortality rates worldwide every year. Due to few early symptoms, most patients are diagnosed with advanced stage, and the 5-year survival rates are $<40 \%$. Although the standard taxane/platinum regiment results in a complete response rate of 40-60\% in advanced ovarian cancer patients, relapse occurs in $>70 \%$ of the patients $(1,2)$. Tumor metastasis and invasion play important roles in cancer development, while there is no effective means to determine the degree of metastasis and invasion. Heparanase (HPSE) is encoded by HPSE which located on chromosome 4 (4q21.3) and is the only endonuclease which can degrade the heparan sulfate proteoglycans (HSPGs) in vivo, remodel extracellular matrix (ECM) via depolymerizing HS chains which are covalently attached to the HPSGs. HPSE may regulate angiogenesis, tissue repair and lipid metabolism by releasing HS bound growth factors and enzymes such as basic fibroblast growth factor (bFGF) (3).

Correspondence to: Professor Li Li, Department of Gynecologic Oncology, Affiliated Tumor Hospital of Guangxi Medical University, 71 Hedi Road, Nanning, Guangxi 530021, P.R. China E-mail: lili@gxmu.edu.cn

Key words: heparanase, ovarian cancer, tumor metastasis, biological behavior, clinical significance
It has been proved that HPSE is overexpressed in several types of malignant cancer such as liver cancer, pancreas cancer, endometrial carcinoma and ovarian cancer, and is associated with tumor invasion and metastasis (4-6). Experiments in vitro also revealed that HPSE plays important roles in tumor metastasis, and it may be a potential biomarker for the determination of metastasis status $(7,8)$. In this study, the relationship between the clinicopathological factors and the expression of HPSE in ovarian cancer and the amounts of HPSE in serum were analyzed. Overexpression and gene silencing of HPSE in epithelial ovarian cancer cells were performed to study its effects on the biological behavior of tumor cells. This study illustrated the biological functions of HPSE in tumor metastasis, and provided useful information for the diagnosis of tumor metastasis and clinical treatment of ovarian cancer.

\section{Materials and methods}

Sample. All blood samples were collected either from patients who were diagnosed with malignant/benign ovarian tumors, or from healthy females undergoing routine physical examinations at the Department of Gynecologic Oncology, Affiliated Tumor Hospital of Guangxi Medical University. The malignant group consisted of 177 cases of ovarian cancer, including 109 cases of serous cystadenocarcinoma, 54 of mucinous cystadenocarcinoma, and 14 of undifferentiated carcinoma according to the criteria of the World Health Organization (WHO, 1973). Among them, 81 cases were stages I-II and 96 cases were stages III-IV in accordance with FIGO standards (2004). The age of patients was 26-67 years (average, 44.6 years), and all patients were followed up for 2.4-62.16 months (mean, 41.10 months). The benign group consisted of 101 cases, including 59 of serous cystadenoma, 42 of ovarian teratoma, and the patients for these samples were aged 14-64 years (average, 35.6 years). The control group consisted of 49 healthy female aged 25-53 years (average, 43.4 years). All blood samples were obtained prior to any treatments, and $2 \mathrm{ml}$ of blood was centrifuged at 3,000 rpm for $5 \mathrm{~min}$, and the supernatants were kept at $-80^{\circ} \mathrm{C}$.

All ovarian samples were collected from patients after surgery in the Department of Gynecologic Oncology, Affiliated Tumor Hospital of Guangxi Medical University and were diagnosed by pathologists. Of the 57 cases of malignant 


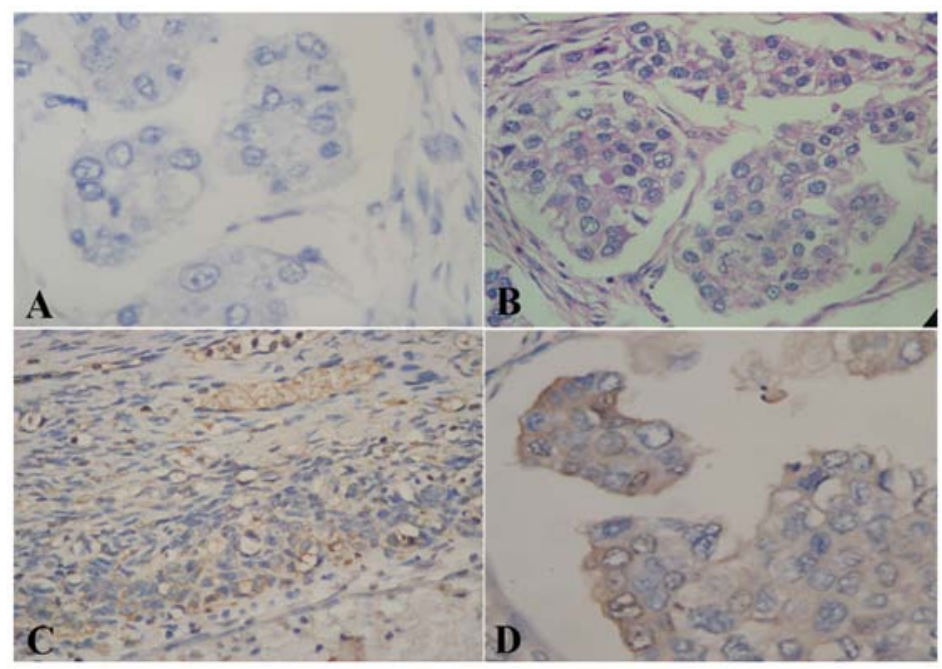

Figure 1. Immunohistochemical staining images of HPSE expression in different ovarian tissues (x40). (A) PBS instead of HPSE primary antibody was used as negative control (-); (B) expression of HPSE in malignant ovarian tumors (-); (C) expression of HPSE in normal ovarian tissues (-); (D) expression of HPSE in low differentiated ovarian adenocarcinoma $(+)$.

ovarian tumors, 30 were serous cystadenocarcinoma, 12 were mucinous cystadenocarcinoma, and 15 were low differentiated adenocarcinomas according to the WHO criteria; 26 cases were stages I-II and 31 were stages III-IV in accordance with FIGO standards. All patients for those 57 samples ranged in age from 28 to 73 years (average, 47.4 years). There were 23 cases of benign ovarian tumors, including 10 of serous cystadenoma, 7 of mucinous cystadenoma and 6 of ovarian teratoma, and the patients were aged 18-73 years (average, 43.4 years). There were 22 cases of normal ovarian tissues excised when the patients underwent the myomectomy or total hysterectomy, following receipt of informed consent and were confirmed to be normal by a pathologist; the patients were aged 48-67 years (average, 58.7 years). The ovarian sample for cDNA cloning of HPSE was a mucinous adenocarcinoma diagnosed by pathologists. The study was endorsed by the Ethics Committee of the Guangxi Medical University. All patients received an explanation concerning the aims of the study and provided signed informed consent. All samples were collected from primary lesions during surgery, and stored in a liquid nitrogen tank. The stored samples were then ready for mRNA isolation and histopathological examination.

Vectors and cells. pcDNA3.1 expression vector (Invitrogen), pEGFP-N1 vector (Clontech) and E Coli DH-5 $\alpha$ were laboratory kept vectors. Ovarian cancer cells including A2780, SKOV3 and HO-8910 were purchased from Shanghai Institute of Cellular Biology of Chinese Academy of Sciences. Cells including HO-8910pm, CBP-resistant SKOV3 and DDP-resistant SKOV3 were established in our laboratory.

Primers. Primers were designed according to the nucleotide sequence of HPSE mRNA which had been deposited in the GenBank database (NM_006665). The gene specific primers were: primers for measurement of HPSE expression in ovarian cancer: forward, 5'-TCCGAGAACACTACCAG-3' and reverse, 5'-GCATCTTAGCCGTCTTTC-3'. $\beta$-actin was used as the control gene: forward, 5'-CTCCATCCTGGCCTCGCTGT-3' and reverse, 5'-GCTGTCACCTTCACCGTTCC-3'. Primers for cDNA cloning of HPSE: forward, 5'-CCGCTCGAGATG CTGTGCGCTCGAAG-3' and reverse, 5'-CCGGAATTCAT TTTCAGATGCAAGCAG-3'.

Six siRNAs for HPSE silencing approach were: 1) pGPU6/ GFP/Neo-heparanase-548: GGAGAAGTTACGGTTGGA ATG; 2) pGPU6/GFP/Neo-heparanase-640: GCTCTGTAGA TGTGCTATACA; 3) pGPU6/GFP/Neo-heparanase-1222: GCTTTATGTGGCTGGATAAAT; 4) pGPU6/GFP/ Neo-heparanase-1556: GCAAGTGGATAAATACCTTCT; 5) positive control (pGPU6/GFP/Neo-shGAPDH): GTAT GACAACAGCCTCAAG; 6) Negative control (pGPU6/GFP/ Neo-shNC): GTTCTCCGAACGTGTCACGT.

Measurement of HPSE expression. Protein expression of HPSE was measured by streptavidin-biotin complex assay (SABC). The SABC kit was purchased from Wuhan Boster Bio-Engineering Inc., (Wuhan, China). Anti-HPSE polyclonal antibody was purchased from Santa Cruz Inc. The images were reviewed in a blinded manner by two experienced pathologists. The determination of staining intensity was as follows: the cytoplasm of ovarian cancer cells exhibiting brown granular staining (Fig. 1D) was considered positive staining and samples showing the absence of staining (Fig. 1A-C) were considered negative. The intensity of protein expression was related identically to the rate of positive cells. The cells with cytoplasmic or membranous staining showing dark brown granules were determined to exhibit strong positivity (score 3). Cells stained light brown indicated weakly positive (score 1) staining, and cells with no brown granules were scored 0 . Intensity between strong positivity and weak positivity was considered as medium positive (score 2) intensity. The positive staining of cells was determined by the number of positive cells vs. the number of total cells at high magnification. A percentage of $<5 \%$ cells was scored as $0 ; 6-25 \%, 1 ; 26-50 \%, 2 ; 51-75 \%, 3$ and $>75 \%, 4$. The product of the staining intensity and the positive rate of cells in each field was determined to be the immunity score, and average score of 5 visions in each section was the final immunity score. 
A final immunity score ranging from $0-2$ was determined as negative and a score $\geq 2$ was determined as positive.

The concentration of HPSE in serum was measured using enzyme-linked immunosorbent assay (ELISA) in accordance with the manufacturer's instructions.

$R T-P C R$. The transcript expression of HPSE was measured by RT-PCR. Total RNA was isolated using TRIzol Reagents (Gibco, USA) and first strand cDNA was synthesized using M-MuLV system (MBI-Fermentas) from $2 \mu \mathrm{g}$ RNA. Primers were designed according to the nucleotide sequence that had been deposited in the GenBank database. Polymerase chain reaction amplification was performed with the following protocol: initial denaturation at $94^{\circ} \mathrm{C}$ for $5 \mathrm{~min}$, followed by a variable number of 35 cycles, $94^{\circ} \mathrm{C}$ for $30 \mathrm{sec}$, specific annealing temperature for $30 \mathrm{sec}$, elongation at $72^{\circ} \mathrm{C}$ for $45 \mathrm{sec}$, and then a final elongation at $72^{\circ} \mathrm{C}$ for $5 \mathrm{~min}$. PCR products were visualized on $2 \%$ agarose gels containing ethidium bromide and photographed using imaging system. PCR products of HPSE were purified and sequenced.

Establishment of stable HPSE up- and downregulated ovarian cell lines. The stable HPSE upregulated cell line was established as follows: cDNA of HPSE cloned from tissue of epithelial ovarian cancer by PCR was sub-cloned into BglII sites of pGEM-T Easy Vector (Promega) for sequencing. Then the XholI and EcoRI fragments were inserted into XholI and EcoRI-digested pcDNA3.1 vector to generate HPSE recombinant expression system, and the insert was confirmed by sequencing. The pcDNA3.1-HPSE was transfected into A2780 cells using Lipofectamine 2000 (Invitrogen, USA). Individual clones were screened for G418-based induction. RT-PCR and western blotting were performed to measure the HPSE expression.

The stable HPSE silencing cell line was established as follows: transfection was performed at $\sim 80 \%$ confluency in six-well plates (Corning, NY, USA) using Lipofectamine 2000 (Invitrogen) according to the manufacturer's instructions. Four purified pGPU6/GFP/Neo-shRNA expression vectors which contained the HPSE shRNA insert and two control vectors were transfected into SKOV3 cells with $10 \mu \mathrm{l}$ of Lipofectamine 2000 reagent. After $48 \mathrm{~h}$ of transfection, RT-PCR and western blotting were performed to assess the efficiency of HPSE knockdown.

Methods to determine the cell biological behaviors. Cell growth inhibition was determined by MTT assay, with 3 replications (4); cell proliferation rate was determined using the colony forming assay as previously reported (5); cell cycle was detected by flow cytometry, and the proportion of cells in the G1, G2 and S phases of cell cycle was analyzed using multicycle software. Cell invasion in vitro was measured by Matrigel invasion, and the kit was purchased from Biological Centre of Peking University (Beijing, China); cell migration in vitro was measured by Transwell migration, and the kit was purchased from Corning Costar (Cambridge, MA, USA); cell adhesion in vitro was measured by Adhesion assay, and the kit was purchased from Biological Centre of Peking University. All steps were carried out in accordance with the manufacturer's instructions.

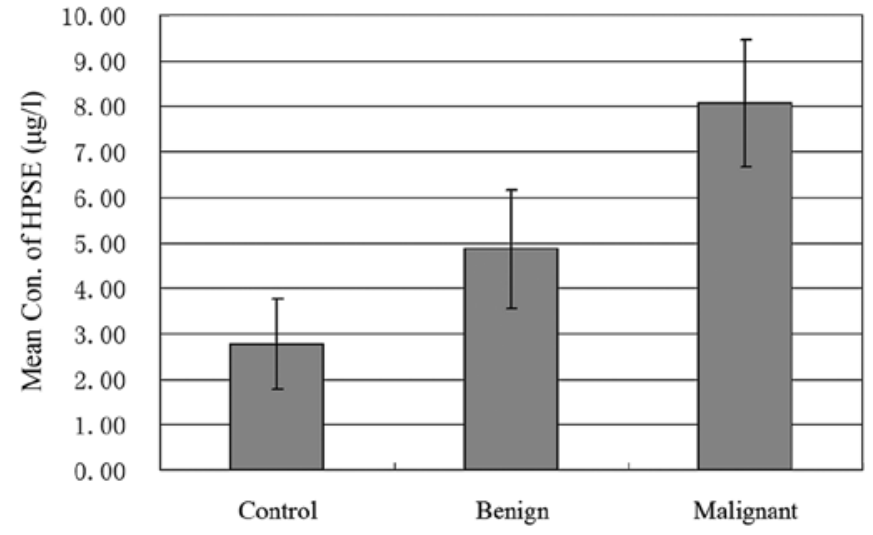

Figure 2. The concentration of HPSE in serum of healthy patients and patients with malignant and benign ovarian tumors.

Statistical analysis. The data were analyzed by SPSS13.0 software. The results of ELISA are presented as the means \pm SD. The measurement data were analyzed using one-way ANOVA, complemented with Kruskal-Wallis tests. The statistical data were analyzed with the Chi-square tests; comparison within groups was analyzed using t-test. $\mathrm{p}<0.05$ was considered to indicate a statistically significant difference. The prognosis was analyzed using the Cox model.

\section{Results}

Serum concentration of HPSE and its relationship with clinicopathological factors in ovarian cancer. As shown in Fig. 2, the concentration of HPSE in serum of patients with malignant ovarian tumors was visibly higher than that in the serum of patients with benign ovarian tumors and controls $(p=0.0)$, but no statistical difference between the benign group and controls was observed. Further study revealed that the amount of HPSE in ovarian cancer patients with distant metastasis was notably higher than the amount in patients with no distant metastasis $(p=0.042)$, but no relationship between serum concentration of HPSE and lymphatic, pelvic and peritoneal metastasis was observed (as shown in Table I). Moreover, the concentration of HPSE in serum of patients with serous, mucinous and undifferentiated cancer showed no statistical difference, suggesting there was no direct relationship between serum concentration of HPSE and histological type, although the serum concentration of HPSE in patients with poorly differentiated and stages III-IV cancer were significantly higher than the concentrations in well differentiated and stages I-II cancer $(p=0.035$ and 0.000 , respectively).

In addition, the diagnostic value of serum HPSE concentration prior to surgery was also evaluated. As shown in Table II, when the serum CA125 concentration was $35 \mathrm{U} / \mathrm{ml}$, the sensitivity for prognosis was $75.7 \%$, the specificity for prognosis was $69.5 \%$, the area under the ROC curve was 0.776 , the standard deviation was 0.030 , and the $95 \%$ confidence interval was $0.717,0.836(\mathrm{p}=0.000)$; in comparison, when the serum HPSE concentration was $4.60 \mathrm{ng} / \mathrm{ml}$, the sensitivity was $76.3 \%$, the specificity was $55.9 \%$, the area under the ROC curve was 0.685 , the standard deviation was 0.031 , and the $95 \%$ confidence interval was $0.700,0.823(\mathrm{p}=0.000)$. These 
Table I. The relationship between serum concentration of HPSE and clinicopathological factors.

Serum concentration of

Clinicopathological factors

No. of samples

HPSE $(\mu \mathrm{g} / \mathrm{l})($ mean \pm STDEV)

Pathological type

Epithelial cancer

177

$8.05 \pm 2.05$

Serous cancer

109

$8.10 \pm 2.02$

Mucinous cancer

54

$7.97 \pm 2.14$

Undifferentiated adenocarcinomas

14

$8.06 \pm 2.17$

Differentiation grade

High-medium differentiated cancer $\quad 29$

$7.20 \pm 2.51$

Low differentiated cancer

148

$8.22 \pm 1.92$

FIGO stage

Stages I-II cancer

62

$7.21 \pm 2.05$

Stages III-IV cancer

115

$8.51 \pm 1.92$

Metastasis

Intra-abdominal lymph node metastasis (+)

$8.11 \pm 2.09$

Intra-abdominal lymph node metastasis (-)

Pelvic metastasis $(+)^{\mathrm{a}}$

125

Pelvic metastasis (-)

52

$8.11 \pm 2.09$

$8.00 \pm 2.04$

Peritoneal metastasis $(+)^{\mathrm{b}}$

115

$8.20 \pm 2.19$

Peritoneal metastasis (-)

62

$7.29 \pm 1.761$

Distant metastasis (+) ${ }^{\mathrm{c}}$

32

$8.63 \pm 1.69$

Distant metastasis (-)

$7.93 \pm 2.11$

$(+)$, positive, (-), negative. ${ }^{\text {aT }}$ The cancer spread to the uterus and/or fallopian tubes and/or other pelvic tissues. ${ }^{\text {b}}$ The cancer extended to liver surface and/or spleen and/or small intestine. ${ }^{~ T h e ~ c a n c e r ~ e x t e n d e d ~ t o ~ l u n g ~ a n d / o r ~ b r a i n ~ a n d / o r ~ b o n e ~ a n d / o r ~ l i v e r ~ p a r e n c h y m a . ~}$

Table II. Comparison of diagnosis value of HPSE and CA125 in serum concentration.

\begin{tabular}{|c|c|c|c|c|c|c|c|}
\hline & $\begin{array}{l}\text { Area under } \\
\text { ROC curve }\end{array}$ & $\begin{array}{c}\text { Sensitivity } \\
\%\end{array}$ & $\begin{array}{c}\text { Specificity } \\
\%\end{array}$ & $\begin{array}{c}\text { Missed diagnosis } \\
\text { rate } \%\end{array}$ & $\begin{array}{c}\text { Misdiagnosis } \\
\text { rate } \%\end{array}$ & $\begin{array}{c}\text { Positive } \\
\text { likelihood ratio }\end{array}$ & $\begin{array}{c}\text { Negative } \\
\text { likelihood ratio }\end{array}$ \\
\hline HPSE & 0.685 & $\begin{array}{c}69.6 \\
(80 / 115)\end{array}$ & $\begin{array}{c}67.7 \\
(42 / 62)\end{array}$ & $\begin{array}{c}30.4 \\
(35 / 115)\end{array}$ & $\begin{array}{c}32.3 \\
(20 / 62)\end{array}$ & 2.1 & 0.5 \\
\hline CA125 & 0.776 & 75.7 & 69.5 & 24.3 & 30.5 & 2.5 & 0.3 \\
\hline
\end{tabular}

results revealed that all the parameters for diagnosis with HPSE concentration were similar or close to the parameters for diagnosis with CA125 concentration, indicating that HPSE may be a potential biomarker for ovarian cancer diagnosis.

Positive expression of HPSE in ovarian cancer and its relationship with clinicopathological factors. As shown in Table III, the positive expression rates of HPSE at both the mRNA and protein levels in malignant ovarian tumors were significantly higher than the rates in benign tumors and controls ( $\mathrm{p}=0.013$ and 0.007 , respectively), while no statistically significant difference was observed between the rates in benign tumors and controls. As shown in Table IV, further study showed that the positive expression rate of HPSE in malignant ovarian cancer had no direct correlations with histological type, but was closely related to differentiation grade and stages. Compared to the rates in well differentiated and stages III-IV cancer, the positive expression rates of HPSE at both the mRNA and protein levels were clearly upregulated in low differentiated and stages I-II cancer $(\mathrm{p}=0.025$ and $0.014 ; 0.013$ and 0.001 , respectively), and the rate in ovarian cancer patients with distant metastasis was notably higher than that in patients with no distant metastasis $(\mathrm{p}=0.027$ and 0.003$)$, although no relationship between the rates and lymphatic, pelvic and peritoneal metastasis was observed.

The positive expression of HPSE was also closely associated with prognosis in ovarian cancer. Among all the factors involved in prognosis such as age, histological type, differentiation grade, FIGO stage, lymphatic metastasis, peritoneal metastasis, distant metastasis, peritoneal fluid and tumor residues, we found that tumor residue, lymphatic metastasis, distant metastasis, mRNA expression of HPSE and the posi- 
Table III. The expression of HPSE at both the mRNA and protein levels in ovarian tumors and controls.

\begin{tabular}{lccc}
\hline Sample group & No. of samples & $\begin{array}{c}\text { mRNA expression of } \\
\text { HPSE (mean } \pm \text { STDEV) }\end{array}$ & $\begin{array}{c}\text { Protein expression } \\
\text { rate of HPSE (\%) }\end{array}$ \\
\hline Controls & 22 & $0.06 \pm 0.120$ & 18.2 \\
Benign ovarian tumors & 23 & $0.077 \pm 0.146$ & 30.4 \\
Malignant ovarian tumors & 57 & $0.176 \pm 0.121$ & 73.7 \\
\hline
\end{tabular}

Table IV. The positive expression of HPSE in ovarian cancer and its relationship to clinicopathological factors.

\begin{tabular}{|c|c|c|c|}
\hline Clinicopathological factors & $\begin{array}{l}\text { No. of } \\
\text { samples }\end{array}$ & $\begin{array}{c}\text { Serum concentration of } \\
\text { HPSE }(\mu \mathrm{g} / \mathrm{l})(\text { mean } \pm \text { STDEV })\end{array}$ & $\begin{array}{c}\text { Protein expression rate } \\
\text { of } \operatorname{HPSE}(\%)\end{array}$ \\
\hline \multicolumn{4}{|l|}{ Pathological type } \\
\hline Serous cancer & 30 & $0.166 \pm 0.172$ & 73.3 \\
\hline Mucinous cancer & 12 & $0.146 \pm 0.184$ & 75.0 \\
\hline Undifferentiated adenocarcinomas & 15 & $0.176 \pm 0.127$ & 73.3 \\
\hline \multicolumn{4}{|l|}{ Differentiation grade } \\
\hline High-medium differentiated cancer & 15 & $0.105 \pm 0.107$ & 46.7 \\
\hline Low differentiated cancer & 42 & $0.287 \pm 0.164$ & 83.3 \\
\hline \multicolumn{4}{|l|}{ FIGO stages } \\
\hline Stages I-II cancer & 26 & $0.031 \pm 0.073$ & 53.8 \\
\hline Stages III-IV cancer & 31 & $0.238 \pm 0.176$ & 90.3 \\
\hline \multicolumn{4}{|l|}{ Metastasis } \\
\hline Intra-abdominal lymph node metastasis $(+)$ & 23 & $0.223 \pm 0.186$ & 86.9 \\
\hline Intra-abdominal lymph node metastasis (-) & 34 & $0.123 \pm 0.165$ & 64.7 \\
\hline Pelvic metastasis $(+)^{\mathrm{a}}$ & 46 & $0.166 \pm 0.187$ & 73.9 \\
\hline Pelvic metastasis (-) & 11 & $0.148 \pm 0.169$ & 72.7 \\
\hline Peritoneal metastasis $(+)^{\mathrm{b}}$ & 31 & $0.201 \pm 0.180$ & 83.9 \\
\hline Peritoneal metastasis (-) & 26 & $0.116 \pm 0.167$ & 61.5 \\
\hline Distant metastasis $(+)^{c}$ & 12 & $0.174 \pm 0.193$ & 91.7 \\
\hline Distant metastasis (-) & 45 & $0.101 \pm 0.173$ & 68.8 \\
\hline
\end{tabular}

$(+)$, positive, $(-)$, negative. ${ }^{a}$ The cancer spread to the uterus and/or fallopian tubes and/or other pelvic tissues. ${ }^{\text {b}}$ The cancer extended to liver surface and/or spleen and/or small intestine. ${ }^{\circ}$ The cancer extended to lung and/or brain and/or bone and/or liver parenchyma.

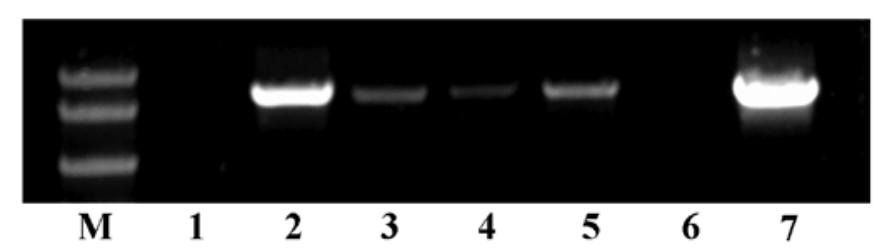

Figure 3. The expression of HPSE in different ovarian cancer cells measured by RT-PCR. Lane M, DNA marker; lane 1, A2780 cells; 2, HO-8910 cells; 3, HO-8910pm cells; 4, HO-8910-175-1 cells; 5, CBP-resistant SKOV3; 6, DDP-resistant SKOV3 cells; 7, normal SKOV3 cells.

tive protein expression rate of HPSE were closely associated with prognosis in ovarian cancer according to COX model analysis (as shown in Table V) $(\mathrm{p}=0.039,0.031,0.031,0.028$ and 0.01 , respectively).
Expression of HPSE in ovarian cancer cells and its effects on cell biological behaviors

cDNA cloning of HPSE and its expression in different ovarian cancer cells. cDNA of HPSE was cloned from tissue of epithelial ovarian cancer by PCR, and the length was $\sim 1,600$ bp in accordance with standard DNA marker, consistent with the theoretical length of HPSE. The cDNA was sub-cloned into pcDNA3.1 vector and sequenced to be $100 \%$ identical to the sequence of HPSE already deposited in GenBank. The expression of HPSE in ovarian cancer cells varied fairly. As shown in Fig. 3, RT-PCR results indicated that HPSE was expressed in HO-8910pm, HO-8910-178-1 and CBP-resistant SKOV3 ovarian cancer cells, and was highly expressed in HO-8910 and normal SKOV3 cells, while no expression was detected in A2780 and DDP-resistant SKOV3 cells. Based on these results, the A2780 cells with no HPSE expression, and SKOV3 cells with strong HPSE expression were selected for subsequent studies. 
Table V. COX model analysis of prognosis related factors in ovarian cancer.

\begin{tabular}{|c|c|c|c|c|c|c|c|c|}
\hline \multirow{2}{*}{$\begin{array}{l}\text { Clinicopathological } \\
\text { factors }\end{array}$} & \multirow[b]{2}{*}{ B } & \multirow[b]{2}{*}{ SE } & \multirow[b]{2}{*}{ Wald } & \multirow[b]{2}{*}{ df } & \multirow[b]{2}{*}{ Sig } & \multirow[b]{2}{*}{$\operatorname{Exp}(B)$} & \multicolumn{2}{|c|}{$\begin{array}{l}95.0 \% \mathrm{CI} \text { for } \\
\operatorname{Exp}(\mathrm{B})\end{array}$} \\
\hline & & & & & & & Lower & Upper \\
\hline Histological type & 1.344 & 0.744 & 3.270 & 1 & 0.071 & 3.836 & 0.893 & 16.474 \\
\hline Histological grade & 0.855 & 0.560 & 2.238 & 1 & 0.127 & 2.352 & 0.784 & 7.053 \\
\hline FIGO stage & 0.702 & 0.574 & 1.495 & 1 & 0.221 & 2.018 & 0.655 & 6.217 \\
\hline $\begin{array}{l}\text { HPSE mRNA } \\
\text { expression }\end{array}$ & -4.572 & 2.077 & 4.848 & 1 & 0.028 & 0.010 & 0.000 & 0.065 \\
\hline $\begin{array}{l}\text { Protein positive } \\
\text { expression rate of HPSE }\end{array}$ & 2.318 & 0.906 & 6.552 & 1 & 0.010 & 10.032 & 1.721 & 59.946 \\
\hline Pelvis metastasis & 0.139 & 0.546 & 0.065 & 1 & 0.799 & 1.149 & 0.394 & 3.351 \\
\hline Peritoneal metastasis & 0.461 & 0.544 & 0.717 & 1 & 0.397 & 1.585 & 0.546 & 4.603 \\
\hline Distant metastasis & 0.983 & 0.533 & 3.201 & 1 & 0.039 & 0.449 & 0.521 & 0.901 \\
\hline Lymph node metastasis & 0.361 & 0.547 & 0.617 & 1 & 0.031 & 0.581 & 0.571 & 6.603 \\
\hline Residue & -1.083 & 0.526 & 4.241 & 1 & 0.039 & 0.339 & 0.121 & 0.949 \\
\hline Age & 0.032 & 0.021 & 2.392 & 1 & 0.122 & 1.032 & 0.991 & 1.075 \\
\hline
\end{tabular}

Table VI. Transfection rates of different shRNA in SKOV3 cells.

\begin{tabular}{lcccc}
\hline SKOV3 cells & Total cells (no.) & GFP cells (no.) & Transfection rate & P-value \\
\hline -HPSE-548 & $110.6 \pm 4.25$ & $58.2 \pm 2.63$ & $53.41 \pm 1.03$ & \\
-HPSE-640 & $111.2 \pm 4.35$ & $56.2 \pm 5.95$ & $50.34 \pm 4.40$ & 0.999 \\
-HPSE-1222 & $112.6 \pm 6.77$ & $57.6 \pm 2.27$ & $53.48 \pm 3.42$ & 1.0 \\
-HPSE-1556 & $102.6 \pm 1.91$ & $48.6 \pm 2.69$ & $47.35 \pm 2.44$ & 0.216 \\
-shGAPDH (positive control) & $109.4 \pm 2.56$ & $65.6 \pm 4.55$ & $58.65 \pm 2.86$ & 0.723 \\
-shNC (negative control) & $122.4 \pm 2.42$ & $60.8 \pm 6.91$ & $49.34 \pm 6.00$ & 0.999 \\
\hline
\end{tabular}

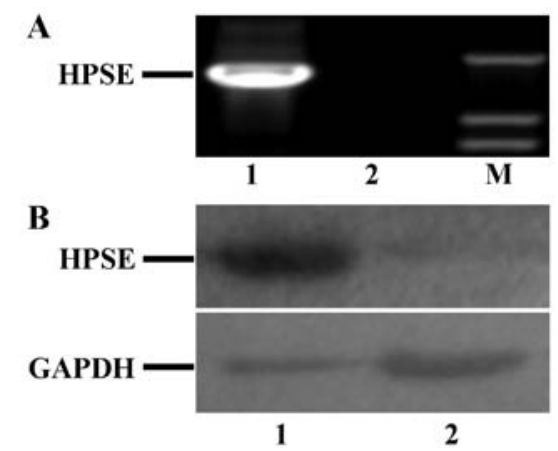

Figure 4. HPSE expression in transfected A2780 cells. (A) Measurement of HPSE expression at mRNA level by PCR; (B) measurement of HPSE expression at protein level by western blotting; lane 1, A2780 cells transfected with pcDNA3.1-HPSE vector; 2, A2780 cells transfected with pcDNA3.1 empty vector; M, DNA marker.

Establishment of stable HPSE up- and downregulated ovarian cell lines. As shown in Fig. 4, compared to the expression in A2780 cells transfected with pcDNA3.1 empty vector, HPSE expression at both the mRNA and protein level was only detected in A2780 cells transfected with pcDNA3.1-HPSE vector. Thus, the A2780 cells transfected with pcDNA3.1HPSE vector was used to study the influence on biological behaviors of tumor cells caused by HPSE.

To gain further insight into the specific function of HPSE, we developed a shRNA interference approach in SKOV3 ovarian cancer cells. Four purified pGPU6/GFP/ Neo-shRNA expression vectors, which contained the HPSE shRNA insert (pGPU6/GFP/Neo-HPSE-548, pGPU6/GFP/ Neo-HPSE-640, pGPU6/GFP/Neo-HPSE-1222, and pGPU6/ GFP/Neo-HPSE-1566) and two control vectors (pGPU6/GFP/ Neo-shNC and pGPU6/GFP/Neo-shGAPDH) were transfected into SKOV3 cells. The transfection rate was measured by GFP cells vs. total cells in one microscope field, each cell with five fields. As shown in Fig. 5 and Table VI, the transfection rates between these six cells had no statistically significant difference. However, the mRNA expression of HPSE in these 6 cells clearly varied. As shown in Fig. 6, HPSE expression at mRNA level in SKOV3 cells transfected with pGPU6/ GFP/Neo-HPSE-1222 was significantly reduced compared to those transfected with pGPU6/GFP/Neo-HPSE-548, pGPU6/ GFP/Neo-HPSE-640, pGPU6/GFP/Neo-HPSE-1556, and two control cells. Thus, pGPU6/GFP/Neo-HPSE-1222 with the greatest silencing efficiency was selected for further study. 
Table VII. Distribution of cells in different phases of cell cycle in different ovarian cancer cells.

\begin{tabular}{lcrr}
\hline & \multicolumn{2}{c}{ No. of cells in cell cycle (mean \pm SD) } \\
\cline { 2 - 4 } Cells & G0 + G1 phase & S phase & G2 + M phase \\
\hline pcDNA3.1-HPSE-A2780 & $56.80 \pm 0.99$ & $26.91 \pm 2.09$ & $16.29 \pm 0.77$ \\
pcDNA3.1-A2780 & $76.61 \pm 0.46$ & $17.93 \pm 0.70$ & $5.47 \pm 0.57$ \\
A2780 & $75.44 \pm 0.38$ & $18.01 \pm 0.55$ & $6.55 \pm 0.63$ \\
pGPU6/GFP/Neo-HPSE-1222-SKOV3 & $87.64 \pm 1.67$ & $8.91 \pm 1.04$ & $3.11 \pm 0.89$ \\
pGPU6/GFP/Neo-shGAPDH-SKOV3 & $74.67 \pm 2.18$ & $14.99 \pm 2.67$ & $10.01 \pm 0.45$ \\
pGPU6/GFP/Neo-shNC-SKOV3 & $77.30 \pm 2.03$ & $12.92 \pm 2.32$ & $8.70 \pm 0.64$ \\
\hline
\end{tabular}
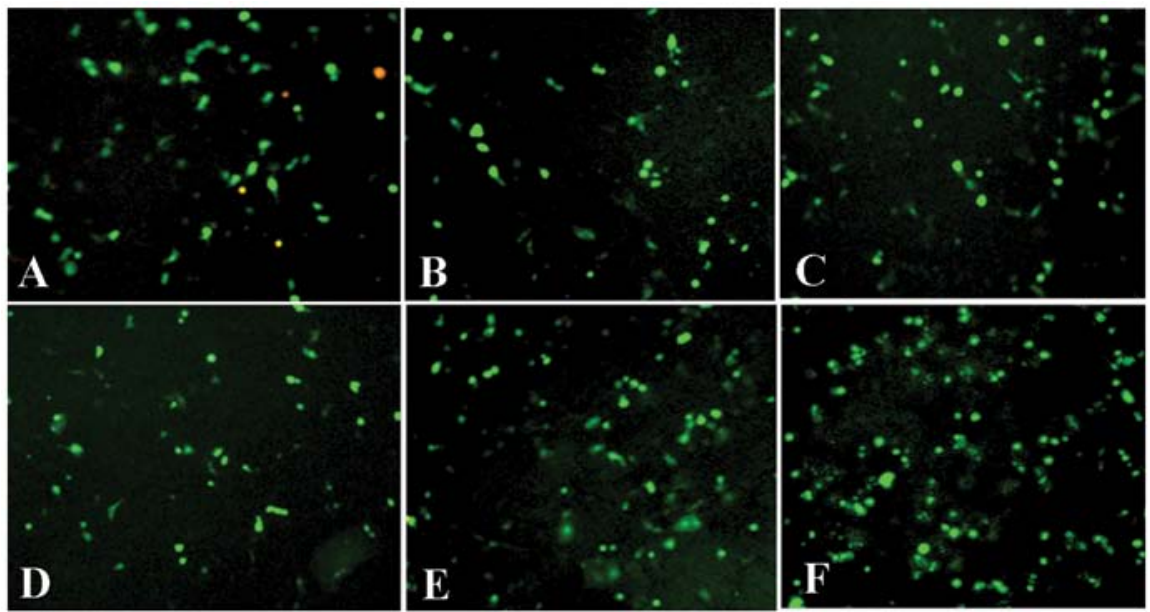

Figure 5. Transfection results of SKOV3 cells with different RNAi vectors. The cells transfected with shRNA was detected under a microscope (x50) after a 48-h incubation. (A) SKOV3 cells transfected with pGPU6/GFP/Neo-HPSE-548; (B) SKOV3 cells transfected with pGPU6/GFP/Neo-HPSE-640; (C) SKOV3 cells transfected with pGPU6/GFP/Neo-HPSE-1222; (D) SKOV3 cells transfected with pGPU6/GFP/Neo-HPSE-1556; (E) SKOV3 cells transfected with pGPU6/GFP/Neo-shGAPDH (positive control); (F) SKOV3 cells transfected with pGPU6/GFP/Neo-shNC (negative control).

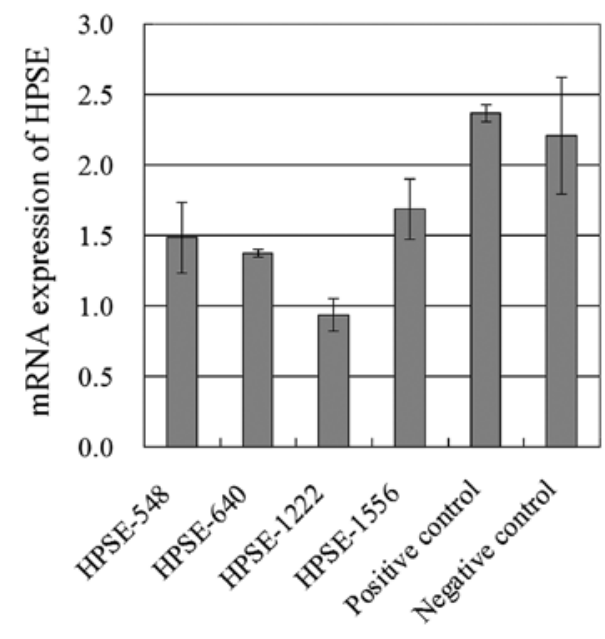

Figure 6. Comparison of HPSE mRNA expression in the four HPSE-silencing cell lines and the two control cell lines. The mRNA expression was measured using RT-qPCR after the cells were incubated for $48 \mathrm{~h}$.

SKOV3 cells transfected with pGPU6/GFP/Neo-HPSE-1222, pGPU6/GFP/Neo-shGAPDH and pGPU6/GFP/Neo-shNC were incubated in G418 medium for 14 days, and those still with
GFP were identified as stable HPSE silencing cells (as shown in Fig. 7). Western blotting was performed to evaluate inhibition of HPSE protein synthesis. As shown in Fig. 8, HPSE protein was specifically silenced in SKOV3 cells transfected with pGPU6/ GFP/Neo-HPSE-1222 plasmids in comparison with that in control cells.

Effects on biological behavior of ovarian tumor cells mediated by altered expression of HPSE. The stable A2780 cells transfected with pcDNA3.1-HPSE vector in which the HPSE was upregulated, and the stable SKOV3 cells transfected with pGPU6/GFP/Neo-HPSE-1222 vector for which the HPSE expression was downregulated, were used to study the effects of HPSE on biological behaviors. 1) The effects on cell growth: the overexpression of HPSE in A2780 cells clearly accelerated the cell growth compared with the growth speed of normal A2780 cells and pcDNA3.1-A2780 cells $(p=0.003)$, and the silencing of HPSE in SKOV3 cells apparently slowed down the cell proliferation in comparison with that in two controls $(\mathrm{p}=0.001) .2)$ The effects on cell cycle: as shown in Table VII, the percentage of cells in proliferative phase ( $S$ phase $+\mathrm{G} 2$ phase $+\mathrm{M}$ phase) of cell cycle in pcDNA3.1-HPSE-A2780 cells was visibly more than that in pcDNA3.1-A2780 cells ( $\mathrm{p}=0.003$ ), and the percentage of 
Table VIII. The effects on cell invasion, metastasis and adhesion of tumor cells mediated by the expression of HPSE.

Cell invasion Cell metastasis Cell adhesion

Cells

Absorbance (mean $\pm \mathrm{SD})$

\begin{tabular}{llrr}
\hline pcDNA3.1-HPSE- 2780 & $0.477 \pm 0.024$ & $1.101 \pm 0.156$ & $0.728 \pm 0.08$ \\
pcDNA3.1-A2780 & $0.250 \pm 0.081$ & $1.051 \pm 0.124$ & $0.518 \pm 0.079$ \\
A2780 & $0.278 \pm 0.077$ & $1.043 \pm 0.133$ & $0.497 \pm 0.063$ \\
pGPU6/GFP/Neo-HPSE-1222-SKOV3 & $0.690 \pm 0.085$ & $0.569 \pm 0.106$ & $0.753 \pm 0.076$ \\
pGPU6/GFP/Neo-shGAPDH-SKOV3 & $1.126 \pm 0.796$ & $0.709 \pm 0.112$ & $0.532 \pm 0.091$ \\
pGPU6/GFP/Neo-shNC-SKOV3 & $1.091 \pm 0.277$ & $0.711 \pm 0.333$ & $0.5833 \pm 0.119$ \\
\hline
\end{tabular}

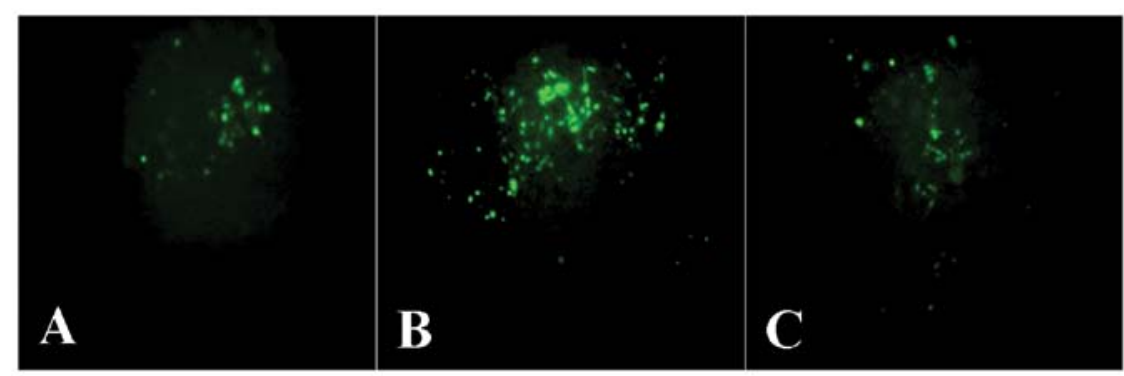

Figure 7. The selection of stable HPSE-silencing SKOV3 cells by G418 after transfection. (A) pGPU6/GFP/Neo-HPSE-1222; (B) pGPU6/GFP/Neo-shGAPDH; (C) pGPU6/GFP/Neo-shNC.

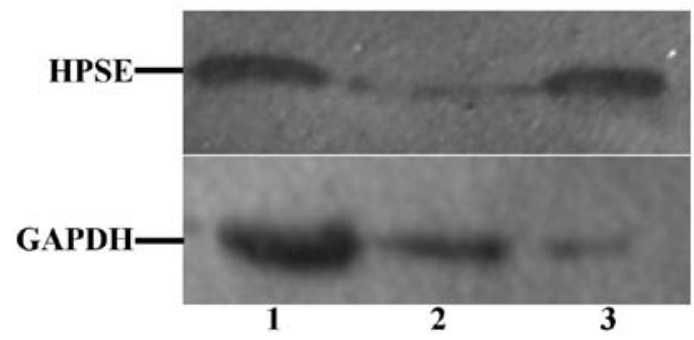

Figure 8. Protein expression of HPSE measured by western blotting in pGPU6/GFP/Neo-HPSE-1222-SKOV3 cells. Lane 1, pGPU6/GFP/NeoshGAPDH-SKOV3 cells; 2, pGPU6/GFP/Neo-HPSE-1222-SKOV3 cells; 3, pGPU6/GFP/Neo-shNC-SKOV3 cells.

cells in the proliferative phase of cell cycle in pGPU6/GFP/ Neo-HPSE-1222-SKOV3 cells was much less than that in control cells ( $\mathrm{p}=0.034$ and 0.015 , respectively). 3 ) The effects on cell invasion, metastasis and cell adhesion: as shown in Table VIII, the ability of cell invasion and adhesion of pcDNA3.1-HPSE-A2780 cells was notably stronger than that of pcDNA3.1-A2780 cells ( $\mathrm{p}=0.003$ and 0.002 ), although no difference was observed in cell metastasis between them. The ability of invasion and adhesion of pGPU6/GFP/Neo-HPSE$1222-$ SKOV3 cells was markedly weaker than that of the two control cells ( $\mathrm{p}=0.015,0.015$ and 0.038 , respectively) and, similarly, no difference was observed in cell metastasis between them. Collectively, these results revealed that the expression of HPSE in ovarian cancer cells had notable effects on biological behaviors of cancer cells, indicating that HPSE might play a role in cancer development in the clinic.

\section{Discussion}

Several studies have focused on the roles of HPSE in tumor invasion and metastasis (9-11). However, the mechanisms of HPSE contributing to tumor invasion and metastasis remain unknown, since those studies were single-factor studies. In this study, based on the measurement of serum HPSE concentration, the expression of HPSE at both the mRNA and protein level in tumors and its effects on biological behaviors of cancer cells, we systematically studied the role of HPSE in tumor invasion and metastasis in ovarian cancer for the first time.

The serum concentration of HPSE and its expression at both the mRNA and protein levels in malignant tumors were clearly higher than in benign tumors and controls. This result was similar to studies previously reported $(12,13)$, suggesting that the overexpression of HPSE might closely relate to cancer development and, therefore, we concluded that either serum HPSE concentration or its expression at mRNA or/and protein levels might be the potential biomarkers for diagnosis in ovarian cancer. Moreover, we observed that serum concentration of HPSE in ovarian cancer patients after surgery was markedly decreased compared with that prior to surgery $(\mathrm{p}=0.023)$, and serum concentration of HPSE in patients with recurrent ovarian cancer was higher that in controls $(\mathrm{p}=0.001)$, suggesting that the serum concentration of HPSE was decreased when the tumor was excised, and increased when the tumor developed again. This finding indicated that HPSE concentration in serum may be a reflection of tumor size and growth state. Previous studies revealed that HPSE was highly expressed in colon cancer, liver cancer, ovarian cancer and 
endometrial carcinoma compared to that in normal controls, respectively (14-17), indicating that HPSE expression is likely associated with tumor invasion, metastasis, neoplastic transformation and cancer development. Collectively, we concluded that the serum concentration of HPSE may be a useful biomarker for evaluation of the surgery effects and prognosis prediction, and may also be useful for condition monitoring in post-operative ovarian cancer patients.

The relationship between clinical pathological factors and the HPSE expression in ovarian cancer was investigated. Compared to that in well differentiated tumors, stages III-IV tumors and tumors without distant metastasis, the positive expression of HPSE at both the mRNA and protein levels in ovarian cancer and the serum concentration of HPSE were all significantly upregulated in poorly differentiated tumors, stages I-II tumors and tumors with distant metastasis, respectively. These results are generally consistent with previous studies reported $(18,19)$.

To gain further insight into the specific function of HPSE in ovarian cancer, we developed the overexpression and silencing approaches to study its effects on biological behaviors in ovarian cancer cells. The results revealed that the altered expression of HPSE led to notable changes in cell growth and cell cycle, indicating that HPSE contributed to cell proliferation in ovarian cancer. This function in ovarian cancer cells is consistent with biological functions of HPSE itself (8).

The altered expression of HPSE had marked effects on the ability of cell adhesion. The cell adhesion ability increased when HPSE was overexpressed, and it decreased when the expression was silenced. The possible mechanism for HPSE worked to cell adhesion might be mediated by uPA. The uPA and tPA were released from HS and were activated after the degradation of basement membrane by HPSE. In turn, the plasminogen binding to cell surface was activated by uPA to dissolve the tumor extracellular matrix. Meanwhile, the uPA was limited and fixed on the tumor cell surface to enhance the affinity of UPA-R with $\mathrm{VN}$ and integrin which were both located on the cell surface. Finally, these cascade reactions led to a gathering and adhesion of those proteins and tumor cells in lesion. Therefore, the decreased adhesive ability of ovarian cancer cells mediated by HPSE were closely related to protease cascade resulting from degradation of basement membrane $(16,20)$.

The expression of HPSE in ovarian cancer cells significantly enhanced the ability of cell invasion, but no apparent change was observed in metastases. This result is consistent with previous studies reporting that HPSE had direct and indirect effects on tumor invasion (21-23). The direct effect of HPSE on cell invasion was that HPSE uniquely degrades heparan sulfate proteoglycans in extracellular matrix (HPSG), causes the degradation of heparin, destroys the extracellular matrix and basement membrane, and eventually promotes the tumor cell invasion.

\section{Acknowledgements}

This study was supported by a grant from the Science and Technology Development Program of Guangxi Science and the Technology Department (no. 2010GXNSFD013053).

\section{References}

1. Shaaban A and Rezvani M: Ovarian cancer: detection and radiologic staging. Clin Obstet Gynecol 52: 73-93, 2009.

2. Chen WQ, Zhang SW, Zou XN and Zhao P: Cancer incidence and mortality in China, 2006. Chin J Cancer Res 23: 3-9, 2011.

3. Hulett MD, Freeman C, Hamdorf BJ, Baker RT, Harris MJ, et al: Cloning of mammalian heparanase, an important enzyme in tumor invasion and metastasis. Nat Med 5: 803-809, 1999.

4. Folkman J and Shing Y: Control of angiogenesis by heparin and other sulfated polysaccharides. Adv Exp Med Biol 313: 355-364, 1992.

5. Mesiano S, Ferrara N and Jaffe RB: Role of vascular endothelial growth factor in ovarian cancer: inhibition of ascites formation by immunoneutralization. Am J Pathol 153: 1249-1256, 1998.

6. Liu S, Li Y, Chen W, Zheng P, Liu T, et al: Silencing glypican-3 expression induces apoptosis in human hepatocellular carcinoma cells. Biochem Biophys Res Commun 419: 656-661, 2012.

7. Jiang G, Zheng L, Pu J, Mei H, Zhao J, et al: Small RNAs targeting transcription start site induce heparanase silencing through interference with transcription initiation in human cancer cells. PLoS One 7: e31379, 2012.

8. Lenaerts L, van Dam W, Persoons L and Naesens L: Interaction between mouse adenovirus type 1 and cell surface heparan sulfate proteoglycans. PLoS One 7: e31454, 2012.

9. Yoneda A, Lendorf ME, Couchman JR and Multhaupt HA: Breast and ovarian cancers: a survey and possible roles for the cell surface heparan sulfate proteoglycans. J Histochem Cytochem 60: 9-21, 2012.

10. Davies EJ, Blackhall FH, Shanks JH, David G, McGown AT, et al: Distribution and clinical significance of heparan sulfate proteoglycans in ovarian cancer. Clin Cancer Res 10: 5178-5186, 2004.

11. Reiland J, Sanderson RD, Waguespack M, Barker SA, Long R, et al: Heparanase degrades syndecan-1 and perlecan heparan sulfate: functional implications for tumor cell invasion. J Biol Chem 279: 8047-8055, 2004.

12. Quiros RM, Rao G, Plate J, Harris JE, Brunn GJ, et al: Elevated serum heparanase-1 levels in patients with pancreatic carcinoma are associated with poor survival. Cancer 106: 532-540, 2006.

13. de Moura JP Jr, Nicolau SM, Stávale JN, da Silva Pinhal MA, de Matos LL, et al: Heparanase-2 expression in normal ovarian epithelium and in benign and malignant ovarian tumors. Int $\mathrm{J}$ Gynecol Cancer 19: 1494-1500, 2009.

14. Peretti T, Waisberg J, Mader AM, de Matos LL, da Costa RB, et al: Heparanase-2, syndecan-1, and extracellular matrix remodeling in colorectal carcinoma. Eur J Gastroenterol Hepatol 20: 756-765, 2008

15. Dong S and Wu XZ: Heparanase and hepatocellular carcinoma: promoter or inhibitor? World J Gastroenterol 16: 306-311, 2010.

16. Davidson B, Shafat I, Risberg B, Ilan N, Trope CG, et al: Heparanase expression correlates with poor survival in metastatic ovarian carcinoma. Gynecol Oncol 104: 311-319, 2007.

17. Inamine M, Nagai Y, Hirakawa M, Mekaru K, Yagi C, et al: Heparanase expression in endometrial cancer: analysis of immunohistochemistry. J Obstet Gynaecol 28: 634-637, 2008.

18. Ginath S, Menczer J, Friedmann Y, Aingorn H, Aviv A, et al: Expression of heparanase, Mdm2, and erbB2 in ovarian cancer. Int J Oncol 18: 1133-1144, 2001.

19. Zhang W, Yang HC, Wang Q, Yang ZJ, Chen H, et al: Clinical value of combined detection of serum matrix metalloproteinase-9, heparanase, and cathepsin for determining ovarian cancer invasion and metastasis. Anticancer Res 31: 3423-3428, 2011.

20. Zetser A, Bashenko Y, Miao HQ, Vlodavsky I and Ilan N: Heparanase affects adhesive and tumorigenic potential of human glioma cells. Cancer Res 63: 7733-7741, 2003.

21. Edovitsky E, Elkin M, Zcharia E, Peretz T and Vlodavsky I: Heparanase gene silencing, tumor invasiveness, angiogenesis, and metastasis. J Natl Cancer Inst 96: 1219-1230, 2004.

22. Shafat I, Ben-Arush MW, Issakov J, Meller I, Naroditsky I, et al: Pre-clinical and clinical significance of heparanase in Ewing's sarcoma. J Cell Mol Med 15: 1857-1864, 2011.

23. Z CZ, Luo C, Yang Z and Wang L: Heparanase participates in the growth and invasion of human U-2OS osteosarcoma cells and its close relationship with hypoxia-inducible factor-1alpha in osteosarcoma. Neoplasma 57: 562-571, 2010. 